Noname manuscript No.

(will be inserted by the editor)

\title{
From a quantum theory to a classical one
}

\author{
A. Coppo · A. Cuccoli - C. Foti · P. Verrucchi
}

Received: date / Accepted: date

\begin{abstract}
We present and discuss a formal approach for describing the quantum to classical crossover based on the group-theoretic construction of generalized coherent states. The method was originally introduced by L. Yaffe 1] in 1982 for tackling large- $N$ quantum field theories, and has been recently used for studying open quantum systems whose environment, while becoming macroscopic, may or may not display a clas-
\end{abstract}

\section{A. Coppo}

Dipartimento di Fisica ed Astronomia Università di Firenze and Istituto Nazionale di Fisica Nucleare, via G. Sansone 1, I-50019 Sesto Fiorentino (Italy)

\section{A. Cuccoli}

Dipartimento di Fisica ed Astronomia Università di Firenze and Istituto Nazionale di Fisica Nucleare, via G. Sansone 1, I-50019 Sesto Fiorentino (Italy)

\section{Foti}

Dipartimento di Fisica ed Astronomia Università di Firenze and Istituto Nazionale di Fisica Nucleare, via G. Sansone 1, I-50019 Sesto Fiorentino (Italy)

P.Verrucchi

Istituto dei Sistemi Complessi-CNR, Dipartimento di Fisica ed Astronomia Università di Firenze, and Istituto Nazionale di Fisica Nucleare, via G. Sansone 1, I-50019 Sesto Fiorentino (Italy) sical behaviour [2,3, 4,5, Referring to these recent developments, in this paper we provide the essential elements of Yaffes's approach in the framework of standard quantum mechanics, so as to clarify how the approach can be used without referring to quantum field theory. Moreover, we address the role played by a possible global symmetry in making the large- $N$ limit of the original quantum theory to flow into a formally well defined classical theory, and we specifically consider the quantum-to-classical crossover of angular momentum. We also give details of a paradigmatic example, namely that of $N$ free one-dimensional spinless particles. Finally, we discuss upon the foundational requirement that any classical description should ultimately be derived from an underlying quantum theory, that however is not, and should never be confused with, the one obtained via some quantization procedure of the classical description itself.

Keywords Quantum-Classical crossover . Open Quantum Systems 


\section{Introduction}

Progresses in quantum technologies have recently made necessary to deeply understand the relation between macroscopic objects that behave according to a classical theory, and the quantum world of microscopic systems, in order to find the best strategies for using, interacting, and exerting control upon small and fragile quantum devices. Key to this understanding is a formal description of the so called quantum to classical crossover, implying the possibility of connecting the geometrical structure of classical physics with the algebraic one featured by quantum mechanics. Some powerful tools in this framework can be found in the literature relative to the so called large- $N$ Quantum Field Theories: although they cannot be straightforwardly used in different settings, such as those typically arising in the analysis of open quantum systems, where the system undergoing the above crossover is just the big partner of a small quantum object, they are versatile enough to be adapted and turn very useful even in these frameworks. In particular, the way L. Yaffe [1] in 1982 tackled some large- $N$ quantum field theories, has demonstrated very powerful and has been recently used for studying open quantum systems whose environment, while becoming macroscopic, may or may not display a classical behaviour 2 , 3, 4,5]. In this paper, after providing the essential elements of Yaffes's approach in the framework of standard quantum mechanics, we elaborate upon the role of the global symmetry, whose presence in the original quantum theory turns out to be a primary requirement to ensure that its large- $N$ limit is a well defined classical theory. The practical implementation of the general abstract approach is described in detail for two specific examples: the quantum-to-classical crossover of angular momentum, and the deduction of the classical limit of a system made of $N$ free one-dimensional spinless particles. The structure of the paper is as follows: In Sec2 we introduce the Generalized Coherent States (GCS, which are essential in Yaffe's procedure) via the group-theoretical approach, independently developed by Gilmore [6] and Perelomov [7] in 1972. Following Ref. [8] we describe the algebraic procedure to construct GCS starting from the knowledge of the dynamical group of the system. In particular, we show how to construct GCS for systems associated to one of the two real forms of the Lie group $S L(2, \mathbb{C})$, namely the non-compact one $S U(1,1)$, whose proper GCS are the so called Pseudo-spin Coherent States (PCS). In Sec 3 we identify the conditions ensuring that a quantum theory has a well defined classical limit, while in Sec4 we consider a specific case to show that such limit can be obtained by increasing the number of degrees of freedom $N$ of the original quantum theory, i.e. when the system it describes becomes macroscopic, as briefly discussed in the last concluding section.

\section{Generalized Coherent States}

Any quantum theory $\mathcal{Q}$ can be defined in terms of an algebra, possibly a Lie algebra $\mathfrak{g}$, and a Hilbert space $\mathcal{H}$, which is the carrier space of an irreducible representation of $\mathfrak{g}$. All the physically relevant operators on $\mathcal{H}$, except for the propagators, are elements of such representation. On the other hand, according to the evolutionpostulate of quantum mechanics, the propagators of $\mathcal{Q}$ are elements of a unitary irreducible representation of the Lie group $\mathcal{G}$ obtained from $\mathfrak{g}$ via a Lie exponential map, for that $\mathcal{G}$ is called "dynamical group". In what follows, for the sake of a lighter presentation, we will most often identify algebras and groups with their respective representations.

Let us now consider a generic quantum system described by a theory $\mathcal{Q}$ such that its Hamiltonian $\hat{H}$ belongs to $\mathfrak{g}$ :

$\hat{H}=H\left(\hat{g}_{i}\right)$, with $\hat{g}_{i} \in \mathfrak{g}$, and $\left[\hat{g}_{i}, \hat{g}_{j}\right]=c_{i j}^{k} \hat{g}_{k}$. 
If we limit our analysis to semisimple Lie algebras (or any algebra admitting a Cartan decomposition), the Cartan basis $\left\{\hat{D}_{i}, \hat{E}_{\alpha}, \hat{E}_{-\alpha}\right\}$ is defined, with

$$
\begin{aligned}
& {\left[\hat{D}_{i}, \hat{D}_{j}\right]=0,\left[\hat{D}_{i}, \hat{E}_{\alpha}\right]=\alpha_{i} \hat{E}_{\alpha},} \\
& {\left[\hat{E}_{\alpha}, \hat{E}_{-\alpha}\right]=\alpha_{i} \hat{D}^{i},\left[\hat{E}_{\alpha}, \hat{E}_{\beta}\right]=C_{\alpha \beta} \hat{E}_{\alpha+\beta},}
\end{aligned}
$$

$\hat{D}_{i}$ Hermitian $\left(\hat{D}_{i}^{\dagger}=\hat{D}_{i}\right)$, and $\hat{E}_{\alpha}$ such that $\hat{E}_{\alpha}^{\dagger}=\hat{E}_{-\alpha}$. The elements $\hat{D}_{i}\left(\hat{E}_{\alpha}\right)$ are dubbed diagonal(shift) operators.

Once a normalized reference state $\left|\Phi_{0}\right\rangle$ in $\mathcal{H}$ is chosen, usually so as to be both an eigenstate of diagonal operators and a maximal weight state, i.e.

$$
\left\{\begin{array}{l}
\hat{D}_{i}\left|\Phi_{0}\right\rangle=d_{i}\left|\Phi_{0}\right\rangle \quad d_{i} \in \mathbb{R} \\
\hat{E}_{\alpha}\left|\Phi_{0}\right\rangle=0 \quad \forall \alpha>0 \\
\left\langle\Phi_{0} \mid \Phi_{0}\right\rangle=1
\end{array}\right.
$$

one can identify the subgroup $\mathcal{F} \subset \mathcal{G}$ that leaves $\left|\Phi_{0}\right\rangle$ invariant up to a phase factor, i.e. $\hat{F} \in \mathcal{F} \rightarrow$ $\hat{F}\left|\Phi_{0}\right\rangle=\left|\Phi_{0}\right\rangle e^{i \varphi(\hat{F})} ;$ this subgroup is called stabilizer of $\mathcal{G}$ with respect to $\left|\Phi_{0}\right\rangle$. Finally, referring to the coset $\mathcal{G} / \mathcal{F}$, the generalized coherent states $|\Omega\rangle$ are defined by:

$\hat{G}\left|\Phi_{0}\right\rangle=\hat{\Omega} \hat{F}\left|\Phi_{0}\right\rangle=\hat{\Omega}\left|\Phi_{0}\right\rangle e^{i \varphi(\hat{F})}:=|\Omega\rangle e^{i \varphi(\hat{F})}$

where

$\hat{G}=\hat{\Omega} \hat{F} \quad \hat{G} \in \mathcal{G}, \hat{F} \in \mathcal{F}, \hat{\Omega} \in \mathcal{G} / \mathcal{F}$.

We notice that GCS are in one-to-one correspondence with the elements $\hat{\Omega}$ of $\mathcal{G} / \mathcal{F}$.

\subsection{Differential structure of $\mathcal{G} / \mathcal{F}$}

According to the "quotient manifold theorem" 9, the coset $\mathcal{G} / \mathcal{F}$ can be associated to a complex manifold $\mathcal{M}$ whose points $\Omega$ are in one-toone correspondence with operators $\hat{\Omega}$ in $\mathcal{G} / \mathcal{F}$, and hence with the states $|\Omega\rangle$. Since the algebra $\mathfrak{g}$ is semisimple, it satisfies the Cartan decomposition in the form $\mathfrak{g}=\mathfrak{f} \oplus \mathfrak{p}$, where $\mathfrak{f}$ is the algebra of $\mathcal{F}$ and $\mathfrak{p}=\xi^{\beta} \hat{E}_{\beta}-\xi^{\beta^{*}} \hat{E}_{-\beta}$ is its orthogonal complement; therefore we can use the coordinates $\left\{\xi^{\beta}, \xi^{\beta^{*}}\right\}$ to write

$\hat{\Omega}=\mathrm{e}^{\left(\xi^{\beta} \hat{E}_{\beta}-\xi^{\beta *} \hat{E}_{-\beta}\right)}, \quad \xi_{\beta} \in \mathbb{C}$.

One can use other coordinate-systems such as, dropping the $\beta$-index for the sake of a lighter notation,

$$
\left\{\begin{array}{l}
\zeta=\xi \frac{\sin \sqrt{\xi^{\dagger} \xi}}{\sqrt{\xi^{\dagger} \xi}} \text { if } \mathcal{M} \text { is compact } \\
\zeta=\xi \frac{\sinh \sqrt{\xi^{\dagger} \xi}}{\sqrt{\xi^{\dagger} \xi}} \text { if } \mathcal{M} \text { is not compact }
\end{array}\right.
$$

or the one yielding a complex projective representation,

$\begin{cases}\tau=\zeta\left(1-\zeta^{\dagger} \zeta\right)^{-\frac{1}{2}} & \text { if } \mathcal{M} \text { is compact } \\ \tau=\zeta\left(1+\zeta^{\dagger} \zeta\right)^{-\frac{1}{2}} & \text { if } \mathcal{M} \text { is not compact }\end{cases}$

\subsection{Metric and measure}

It can be demonstrated [10] that $\mathcal{M}$ is endowed with a natural metric that can be expressed in the $\tau$ coordinates as

$$
\begin{aligned}
& d s^{2}=g_{\alpha \beta} d \tau^{\alpha} d \tau^{\beta^{*}} \text { where } g_{\alpha \beta}:=\frac{\partial^{2} F\left(\tau, \tau^{*}\right)}{\partial \tau^{\alpha} \partial \tau^{\beta^{*}}} \\
& F\left(\tau, \tau^{*}\right)=\log N\left(\tau, \tau^{*}\right), \quad N\left(\tau, \tau^{*}\right)=\langle\tilde{\tau} \mid \tilde{\tau}\rangle \\
& |\tilde{\tau}\rangle=e^{\tau^{\beta}} \hat{E}_{\beta}\left|\Phi_{0}\right\rangle
\end{aligned}
$$

where $|\tilde{\tau}\rangle$ is a non-normalized GCS. This allows one to get information upon the manifold $\mathcal{M}$. Through $d s^{2}$ one can define a canonical volume form on $\mathcal{M}$, i.e. a measure

$d \mu(\Omega)=$ const $\times \operatorname{det}(g) \prod_{\alpha} d \tau^{\alpha} d \tau^{\alpha *}$. 
2.3 Overcompleteness of coherent states

Using $d \mu(\Omega)$, GCS are demonstrated to form an overcomplete set of states on $\mathcal{H}$, providing a continuous resolution of the identity, i.e.

$\hat{\mathbb{I}}=\int_{\mathcal{G} / \mathcal{F}} d \mu(\Omega)|\Omega\rangle\langle\Omega|$.

The prefix "over" in the adjective 'overcomplete' indicates that coherent states are "a lot": in fact, despite being normalized, $\langle\Omega \mid \Omega\rangle=\left\langle\Phi_{0}\right| \hat{G}^{-1}$ $\left\langle\Phi_{0} \mid \Phi_{0}\right\rangle=1, \forall \hat{G} \in \mathcal{G}$, they are not orthogonal,

$$
\begin{aligned}
& \left\langle\Omega \mid \Omega^{\prime}\right\rangle=\left\langle\Phi_{0}\left|\hat{\Omega}^{-1} \hat{\Omega}^{\prime}\right| \Phi_{0}\right\rangle= \\
& =\left\langle\Phi_{0}\left|\hat{G}^{-1} \hat{G}^{\prime}\right| \Phi_{0}\right\rangle \mathrm{e}^{i \varphi}=\left\langle\Phi_{0}\left|\hat{G}^{\prime \prime}\right| \Phi_{0}\right\rangle \mathrm{e}^{i \varphi} \neq 0
\end{aligned}
$$

$\forall \hat{G}, \hat{G}^{\prime}, \hat{G}^{\prime \prime} \in \mathcal{G}$, and $\hat{\Omega}, \hat{\Omega}^{\prime} \in \mathcal{G} / \mathcal{F}$.

\subsection{Symplectic structure}

$\mathcal{M}$ is equipped with a symplectic structure that allows one to identify it as a phase space, possibly the one proper to the classical system into which the original quantum system flows when the classical limit is rigorously performed. The symplectic form on $\mathcal{M}$ has the coordinate representation

$\omega=-i \sum_{\alpha \beta} g_{\alpha \beta} d \tau^{\alpha} \wedge d \tau^{\beta^{*}}$,

and it is used to define the Poisson brackets

$$
\{f, g\}_{P B}:=i \sum_{\alpha \beta} g^{\alpha \beta}\left(\frac{\partial f}{\partial \tau^{\alpha}} \frac{\partial g}{\partial \tau^{\beta^{*}}}-\frac{\partial g}{\partial \tau^{\alpha}} \frac{\partial f}{\partial \tau^{\beta^{*}}}\right) \text {. }
$$

Switching to the $\zeta$ coordinates, and defining $w$ and $v$ via

$$
\zeta_{\beta}=\frac{1}{\sqrt{2}}\left(w_{\beta}-i v_{\beta}\right), \quad \zeta_{\beta}^{*}=\frac{1}{\sqrt{2}}\left(w_{\beta}+i v_{\beta}\right),
$$

one obtains the Poisson brackets in the standard form,

$$
\{f, g\}_{P B}=\sum_{\alpha}\left(\frac{\partial f}{\partial v^{\alpha}} \frac{\partial g}{\partial w^{\alpha}}-\frac{\partial g}{\partial v^{\alpha}} \frac{\partial f}{\partial w^{\alpha}}\right) .
$$

\subsection{Pseudo-spin coherent states}

We end this section by giving an explicit example of GCS construction, namely those relative $\hat{G}$ tक the group $S U(1,1) \sqrt{1}$. Its generators are the set $\left\{\hat{K}_{0}, \hat{K}_{1}, \hat{K}_{2}\right\}$ which spans the $\mathfrak{s u}(1,1)$ algebra

$\left[\hat{K}_{\alpha}, \hat{K}_{\beta}\right]=i \epsilon_{\alpha \beta \gamma} \hat{K}^{\gamma}$

where the indices $\alpha, \beta, \gamma \in\{0,1,2\}$ are raised and lowered with the 3 -dimensional Minkowski metric $\eta_{\alpha \beta}=\operatorname{diag}\{-1,1,1\}$. The Hilbert space of the system is a unitary irreducible representation of $\mathfrak{s u}(1,1)$, which is identified by the so called Bargmann index $k$ :

$\mathcal{H}_{k}=\left\{|k, m\rangle, m \in \mathbb{N} \quad k \in \mathbb{R}^{+}\right\}$,

where $|k, m\rangle$ are the simultaneous eigenstates of $\hat{K}_{0}$ and of the Casimir operator $\hat{K}^{2}=-\hat{K}_{\alpha} \hat{K}^{\alpha}$ such that

$\left\{\begin{array}{l}\hat{K}^{2}|k, m\rangle=k(k-1)|k, m\rangle, \\ \hat{K}_{0}|k, m\rangle=(k+m)|k, m\rangle .\end{array}\right.$

The unitary irreducible representations of $S U(1,1)$ (that are infinite-dimensional since the group is not compact) have been firstly discussed by Bargmann [11] as incidental to his discussion of the Lorentz group. One can find a consolidated review in Ref. [12. In this paper we will only refer to the representations of the group

1 The Lie group $S U(1,1)$ is defined as the group of transformations in the two-dimensional complex plane $\mathbb{C}^{2}$ that leave invariant the Hermitian form $\bar{\psi} \psi:=\psi^{\dagger} \sigma_{3} \psi=\psi_{1}^{\dagger} \psi_{1}-\psi_{2}^{\dagger} \psi_{2}$, where $\psi=\left(\psi_{1}, \psi_{2}\right) \epsilon$ $\mathbb{C}^{2}$ and $\sigma_{3}$ is the third Pauli matrix. This group is isomorphic to $S L(2, \mathbb{R})$ and $S p(2, \mathbb{R})$, and its substantial differences with $S U(2)$ is that it is noncompact and it is not simply connected. We will study an explicit example of a system related to this group in Sec. 3 
$S O(1,2)=S U(1,1) / \mathbb{Z}_{2}$, obtained by Barut and Fronsdal in Ref. [13].

To construct GCS we need a reference state. Given the index- $k$ representation we choose the lowest-weight state i.e. $\left|\Phi_{0}\right\rangle=|k, m=0\rangle$ and we identify the stabilizer subgroup $\mathcal{F}$ by

$e^{i \delta \hat{K}_{0}}|k, 0\rangle=e^{i \delta k}|k, 0\rangle \quad$ with $\quad \delta \in \mathbb{R}$,

and hence $\mathcal{F}=U(1)$. We can now consider the coset $S U(1,1) / U(1)$ to define the pseudo-spin coherent states (PCS)

$|\Omega\rangle=\hat{\Omega}|k, 0\rangle \quad$ with $\hat{\Omega} \in S U(1,1) / U(1)$,

where $\hat{\Omega}$ can be parameterized as:

$\hat{\Omega}=\mathrm{e}^{\xi \hat{K}_{+}-\xi^{*} \hat{K}_{-}}$

with $\hat{K}_{ \pm}=\hat{K}_{1} \pm i \hat{K}_{2}$ shift operators satisfying $\left[\hat{K}_{+}, \hat{K}_{-}\right]=-2 \hat{K}_{0},\left[\hat{K}_{0}, \hat{K}_{ \pm}\right]= \pm \hat{K}_{ \pm}$. Points $\Omega$ on the manifold associated to the coset $S U(1,1) / U$ can be identified by the complex coordinates $\left(\xi, \xi^{*}\right)$; this allows one to express $\hat{\Omega}$, using the standard $(2 \times 2)$ matrix representation 2

$\hat{K}_{+}=\left[\begin{array}{ll}0 & i \\ 0 & 0\end{array}\right] \quad \hat{K}_{-}=\left[\begin{array}{ll}0 & 0 \\ i & 0\end{array}\right] \quad \hat{K}_{0}=\left[\begin{array}{cc}\frac{1}{2} & 0 \\ 0 & -\frac{1}{2}\end{array}\right]$,

by means of the matrix $[\underline{8}$

$\left[\begin{array}{cc}\sqrt{1+\zeta \zeta^{*}} & \zeta \\ \zeta^{*} & \sqrt{1+\zeta \zeta^{*}}\end{array}\right]$,

with $-i \zeta$ defined by Eq. (6) 3 . Introducing "polar" coordinates $(\rho, \phi) \in \mathbb{R} \times[0,2 \pi]$ via

$i \xi=\frac{\rho}{2} \mathrm{e}^{-i \phi}$

Eqs. (6) and (7) define the $\zeta$ - and $\tau$-coordinates as

$\zeta=\sinh \frac{\rho}{2} \mathrm{e}^{-i \phi}, \quad \tau=\tanh \frac{\rho}{2} \mathrm{e}^{-i \phi}$.

2 This representation is finite dimensional and hence not Hermitian.

3 A factor $i$ is needed to define $\zeta$ because the representation (22) is not Hermitian.
Eq. (21) can be written 14 in the $\tau$-coordinates

$\hat{\Omega}=\left(1-|\tau|^{2}\right)^{k} \mathrm{e}^{\tau \hat{K}_{+}}$

so that the natural metric defined in Eq. (8) emerges via

$$
\begin{aligned}
& |\tilde{\tau}\rangle=e^{\tau \hat{K}_{+}}|k, 0\rangle, N\left(\tau, \tau^{*}\right)=\left(1-|\tau|^{2}\right)^{-2 k}, \\
& F\left(\tau, \tau^{*}\right)=-2 k \log \left(1-|\tau|^{2}\right),
\end{aligned}
$$

as

$$
d s^{2}=\frac{2 k}{\left(1-|\tau|^{2}\right)^{2}} d \tau d \tau^{*}=\frac{k}{2}\left(d^{2} \rho+\sinh ^{2} \rho d^{2} \phi\right),
$$

where $|\tilde{\tau}\rangle$ is a non-normalized PCS. Moreover, it is possible to show [14] that the completeness $U$ (1)elation (10) is verified for any $k>1 / 2$ in the form

$\int_{S U(1,1) / U(1)} d \mu_{k}(\tau)|\tau\rangle\langle\tau|=\mathbb{I}$,

with $\quad d \mu_{k}(\tau)=\frac{2 k-1}{\pi} \frac{d \tau d \tau^{*}}{\left(1-|\tau|^{2}\right)^{2}}$.

The manifold associated to $S U(1,1) / U(1)$ is called "Bloch" pseudosphere $P S^{2}$ (see for instance Chap.1 of Ref. [5] for further details).

\section{From a quantum theory to a classical one}

In this section, following Ref. [1, we show how a large- $N$ limit of a quantum theory can formally define a classical dynamics. Let us first specify what makes a theory recognizable as a quantum or a classical one: as mentioned in Sec.2, a quantum theory $\mathcal{Q}$ is defined by:

- a Lie Algebra $\mathfrak{g}$,

- a Hilbert space $\mathcal{H}$ that carries an irreducible representation of $\mathfrak{g}$,

- a Hamiltonian operator $\hat{H} \in \mathfrak{g}$. 
A classical theory $\mathcal{C}$ is instead determined by 4 :

- a manifold $\mathcal{M}$

- a symplectic form on $\mathcal{M}$, which defines Poisson brackets,

- a Hamiltonian function $h_{c l}: \mathcal{M} \rightarrow \mathbb{R}$.

After the above definitions, one can describe a general procedure for realizing a so-called quantumto-classical crossover, which is a formal relation between quantum and classical theories, describing how the first can naturally flow into the latter, possibly when some "quanticity parameter" $\chi \in \mathbb{R}^{+}$tends to zero. The limit $\chi \rightarrow 0$ is dubbed "classical limit" and, in order to exist, certain conditions must be fulfilled, that isolate the minimal structure that the starting quantum theory should possess. These conditions are satisfied by a large class of quantum theories, namely the Large- $N$ quantum theories that feature a global symmetry. If this is the case, $\chi$ is a decreasing function of the number $N$ of degrees of freedom, and $\chi \rightarrow 0$ when $N \rightarrow \infty$. This reveals that many-variables, provided with a global symmetry, lie behind any quantum-toclassical crossover.

3.1 When does a quantum theory have a classical limit?

Consider a quantum theory $\mathcal{Q}_{\chi}$ defined by the Lie algebra $\mathfrak{g}$, the Hilbert space $\mathcal{H}_{\chi}$ and the Hamiltonian $\hat{H}_{\chi}$. Be such theory characterized by some parameter $\chi$ which is assumed to take positive real values, including the limiting $\chi=$ 0 one. Once identified the dynamical group $\mathcal{G}$ of the theory via a Lie exponential map on $\mathfrak{g}$, and its irreducible unitary representation $5 \mathcal{G}_{\chi}$

\footnotetext{
4 More accurately this is the definition of Hamiltonian classical theory, but not all classical theories are Hamiltonian. Anyway in this paper we only consider these ones.

5 Notice that the abstract group $\mathcal{G}$ and its algebra $\mathfrak{g}$ do not depend on $\chi$, which instead enters $\mathcal{G}_{\chi}$ and
}

on $\mathcal{H}_{\chi}$, we can construct the GCS $\left|\Omega_{\chi}\right\rangle$. They will be in one-to-one correspondence with the points $\Omega_{\chi}$ of the manifold $\mathcal{M}_{\chi}$ associated to the coset $\mathcal{G} / \mathcal{F}_{\chi}$, where $\mathcal{F}_{\chi}$ is the stabilizer with respect to a reference state $\left|0_{\chi}\right\rangle \in \mathcal{H}_{\chi}$. For any operator $\hat{A}$ acting on $\mathcal{H}_{\chi}$ one can define the symbol $A\left(\Omega_{\chi}\right)$ by

$A\left(\Omega_{\chi}\right):=\left\langle\Omega_{\chi}|\hat{A}| \Omega_{\chi}\right\rangle, \forall \Omega_{\chi} \in \mathcal{M}_{\chi}$.

As pointed out in Ref.[1], in order to have some control over the limit $\chi \rightarrow 0$, suppose that it is possible to arrange the set of GCS in the equivalence classes

$\left[\left|\Omega_{\chi}\right\rangle\right]_{\sim}:=|\Omega\rangle_{\chi}$

obtained from the equivalence relation

$$
\begin{aligned}
& \left|\Omega_{\chi}\right\rangle \sim\left|\Omega_{\chi}^{\prime}\right\rangle \\
& \text { if } \lim _{\chi \rightarrow 0} A\left(\Omega_{\chi}\right)=\lim _{\chi \rightarrow 0} A\left(\Omega_{\chi}^{\prime}\right)<\infty, \forall \hat{A} \in \mathcal{K},
\end{aligned}
$$

where, in order to ensure that the limit is well defined, $\mathcal{K}$ is a restricted set of operators satisfying

$$
\begin{aligned}
& \lim _{\chi \rightarrow 0} \frac{\left\langle\Omega|\hat{A}| \Omega^{\prime}\right\rangle_{\chi}}{\left\langle\Omega \mid \Omega^{\prime}\right\rangle_{\chi}}:=A\left(\Omega, \Omega^{\prime}\right)_{\chi}<\infty, \\
& \forall \Omega, \Omega^{\prime} \in \mathcal{M}_{\chi} / \sim ;
\end{aligned}
$$

operators in $\mathcal{K}$ will be called classical operators. Since the symbols of classical operators upon GCS that belong to a same class are equal, according to Eq. (32), we will use the notation:

$$
\begin{aligned}
& A_{\chi}(\Omega):=A\left(\Omega_{\chi}\right)=\langle\Omega|\hat{A}| \Omega\rangle_{\chi}, \\
& \forall \Omega \in \mathcal{M}_{\chi} / \sim .
\end{aligned}
$$

It can be demonstrated [1] that, in order for the theory $\mathcal{Q}_{\chi}$ to have a $\chi \rightarrow 0$ limit that corresponds to a classical theory, the following conditions must hold:

its algebra $\mathfrak{g}_{\chi}$ via the $\chi$-dependence of the Hilbert space $\mathcal{H}_{\chi}$. 


\section{1) Irreducibility of $\mathcal{G}_{\chi}$}

As mentioned above, each representation $\mathcal{G}_{\chi}$ of the dynamical group acts irreducibly on the corresponding Hilbert space $\mathcal{H}_{\chi}$. This requirement assures that for each $\chi$ the quantum theory is well defined. Using the Schur's lemma and the invariance of the measure on the $\operatorname{coset} \mathcal{G} / \mathcal{F}_{\chi}$, this assumption implies eq. (10), i.e.

$\mathbb{I}_{\chi}=c_{\chi} \int_{\left(\mathcal{G} / \mathcal{F}_{\chi}\right) / \sim} d \mu(\Omega)|\Omega\rangle\left\langle\left.\Omega\right|_{\chi}\right.$,

where $c_{\chi}$ is a constant depending on the normalization of the group measure and must be computed explicitly. Notice that the measure $d \mu(\Omega)$ does not depend on $\chi$ and hence remains the same as $\chi \rightarrow 0$.

2) Uniqueness of the "Zero" operator

The zero operator $\hat{Z}$ is the only one for which $Z_{\chi}(\Omega)=0 \quad \forall \Omega \in \mathcal{M}_{\chi} / \sim$. As a consequence, two different operators cannot have the same symbol, implying that any operator can be uniquely recovered from its expectation value on GCS, i.e. from its symbols.

3) Exponential decrease of inequivalent coherent states overlap

The overlap between classically inequivalent GCS exponentially decreases as $\chi \rightarrow 0$, i.e.

$\lim _{\chi \rightarrow 0}\left\langle\Omega \mid \Omega^{\prime}\right\rangle_{\chi}=\mathrm{e}^{-\lim _{\chi \rightarrow 0} \frac{\Delta\left(\Omega, \Omega^{\prime}\right)_{\chi}}{\chi}}$

where $\exists \lim _{\chi \rightarrow 0} \Delta\left(\Omega, \Omega^{\prime}\right)_{\chi} \forall \Omega, \Omega^{\prime} \in \mathcal{M}_{\chi} / \sim$ and

$\operatorname{Re} \Delta\left(\Omega, \Omega^{\prime}\right)_{\chi} \begin{cases}>0 & \text { if }|\Omega\rangle_{\chi} \neq\left|\Omega^{\prime}\right\rangle_{\chi} \\ =0 & \text { if }|\Omega\rangle_{\chi}=\left|\Omega^{\prime}\right\rangle_{\chi}\end{cases}$

The result is that, when $\chi \rightarrow 0$ inequivalent coherent states become orthogonal, i.e. distinguishable. As a consequence, the factorization

$\lim _{\chi \rightarrow 0}\left[(A B)_{\chi}(\Omega)-A_{\chi}(\Omega) B_{\chi}(\Omega)\right]=0$

holds for any pair $\hat{A}$ and $\hat{B}$ of classical operators.

\section{4) Classical limit of the Hamiltonian}

The operator $\chi \hat{H}_{\chi}$ is a classical operator. This ensures that the coupling constants in the Hamiltonian are scaled in a manner that maintains sensible dynamics as $\chi \rightarrow 0$, so as to define a meaningful classical limit.

If the hypothesis 1-4 are satisfied there is a phase space on which a classical dynamics can be defined: it is the manifold $\mathcal{M}=\left(\mathcal{M}_{\chi} / \sim\right)_{\chi \rightarrow 0}$ whose points $\Omega$ are in one-to-one correspondence with the GCS classes $\left[\left|\Omega_{\chi \rightarrow 0}\right\rangle\right]_{\sim}:=|\Omega\rangle$, and which can be equipped with the natural metric and the symplectic structure defined by Eqs. (8)-(12). Using the coordinates $w^{\beta}, v^{\beta}$ as in Eq. (14) the classical Hamiltonian turns out to be [1]

$h_{c l}\left(v^{\beta}, w^{\beta}\right)=\lim _{\chi \rightarrow 0} \chi H_{\chi}(\Omega)$.

3.2 Large- $N$ quantum theories: crucial role of global symmetries

All known types of quantum theory $\mathcal{Q}_{N}$ described by $N$ degrees of freedom (dof) and equipped with a global symmetry $\mathfrak{X}(N)$ are found to satisfy the conditions $1-4$, where $\chi=\chi(N)$ is a decreasing function such that $\lim _{N \rightarrow \infty} \chi(N)=0$. The symmetry $\mathfrak{X}(N)$ is called global, for $\mathcal{Q}_{N}$, if the related transformations act on all its $N$ dof. In fact, the existence of the symmetry $\mathfrak{X}(N)$ is crucial, as it is responsible for the possible reduction of dof defining $\mathcal{Q}_{N}$, once it has flowed, for $N \rightarrow \infty$, in the corresponding classical theory. Let us hence show how the symmetry plays its role:

* Saying that a theory $\mathcal{Q}_{N}$ has a certain symmetry implies that all the relevant operators $\hat{A}$ of $\mathcal{Q}_{N}$ satisfy the relation $\mathcal{U} \hat{A} \mathcal{U}^{\dagger}=\hat{A} \quad \forall \mathcal{U} \in$ $\mathfrak{X}(N)$.

* Considering the GCS of $\mathcal{Q}_{N}$ and the symbols defined by Eq. (30), it is hence $A\left(\Omega_{N}\right)=\left\langle\Omega_{N}|\hat{A}| \Omega_{N}\right\rangle=\left\langle\Omega_{N}\left|\mathcal{U} \hat{A} \mathcal{U}^{\dagger}\right| \Omega_{N}\right\rangle=$ $\left\langle\Omega_{N}^{\mathcal{U}}|\hat{A}| \Omega_{N}^{\mathcal{U}}\right\rangle=A\left(\Omega_{N}^{\mathcal{U}}\right)$, where $\left|\Omega_{N}^{\mathcal{U}}\right\rangle:=\mathcal{U}\left|\Omega_{N}\right\rangle$. 
* This suggests to define the equivalence relation:

$\left|\Omega_{N}\right\rangle \sim\left|\Omega_{N}^{\prime}\right\rangle$ if $A\left(\Omega_{N}\right)=A\left(\Omega_{N}^{\prime}\right)$,

for any relevant operator $\hat{A}$, in order to arrange the GCS in the classes $\left[\left|\Omega_{N}\right\rangle\right]_{\sim}:=|\Omega\rangle_{N}$. In this way all the states connected through a symmetry transformation are equivalent.

* In the limit $N \rightarrow \infty$ only the classical operators defined by Eq. (33) clearly remain relevant and, comparing Eq. (39) and (32), one obtains that the points on the classical phase $\mathcal{M}$ are identified by the classes $|\Omega\rangle:=\left[\left|\Omega_{N \rightarrow \infty}\right\rangle\right]_{\sim}$, rather than by the huge number of GCS $\left|\Omega_{N \rightarrow \infty}\right\rangle$.

Finally we remark that not all of the Large$N$ quantum theories flow into classical theories: in order to realize the crossover a global symmetry is needed. In fact, if such a symmetry is not present the theory will remain quantum also for $N \rightarrow \infty$. For instance consider a theory describing a Large- $N$ set of indistinguishable particles: speaking about some global symmetry is clearly meaningless if one cannot distinguish between a variable and another one. Indeed it is well known that the quantum effects in a gas of indistinguishable particles are particularly relevant, especially when its density (for a fixed temperature) is high.

\section{Large $-N$ limit of $O(N)$ vector models}

Consider a $O(N)$ global invariant quantum theory $\mathcal{Q}_{N}$ describing a system of $N$ one-dimensional distinguishable spinless particles: its Hamiltonian acts on the Hilbert space $\mathcal{H}_{N}$ and can be taken as an arbitrary polynomia 6 of the form

$\hat{H}_{N}=N h[\hat{A}, \hat{B}, \hat{C}]$,

6 If $\mathfrak{g}$ is the Lie algebra defining the theory, we consider the Hamiltonian as an element of the universal enveloping algebra $U(\mathfrak{g})=T(\mathfrak{g}) / I$, where $T(\mathfrak{g})=$ $K \oplus \mathfrak{g} \oplus(\mathfrak{g} \otimes \mathfrak{g}) \oplus(\mathfrak{g} \otimes \mathfrak{g} \otimes \mathfrak{g}) \oplus \cdots$ is the tensor algebra of $\mathfrak{g}$ ( $K$ is the field over which $\mathfrak{g}$ is defined) and $I$ is the two-sided ideal over $T(\mathfrak{g})$ generated by elements of the form $\hat{A} \otimes \hat{B}-\hat{B} \otimes \hat{A}-[\hat{A}, \hat{B}]$ with $\hat{A}, \hat{B} \in \mathfrak{g}$. Informally $U(\mathfrak{g})$ is the algebra of the poly- where $\hat{A}, \hat{B}, \hat{C}$ are the basic $O(N)$ invariants:

$$
\left\{\begin{array}{l}
\hat{A}=\frac{1}{2} \sum_{i} \hat{q}_{i}^{2}, \\
\hat{B}=\frac{1}{2} \sum_{i}\left(\hat{q}_{i} \hat{p}_{i}+\hat{p}_{i} \hat{q}_{i}\right), \\
\hat{C}=\frac{1}{2} \sum_{i} \hat{p}_{i}^{2},
\end{array}\right.
$$

with positions $\hat{q}_{i}$ and conjugated momenta $\hat{p}_{i}$ satisfying the canonical commutation relations:

$i\left[\hat{p}_{i}, \hat{q}_{j}\right]=\frac{1}{N} \delta_{i j} \hat{\mathbb{I}}$,

with $i, j=1, \ldots, N$ particle index. Applying the formalism of section 3 with $\chi=1 / N$, as suggested in Ref. [1], one finds the classical limit of the $\mathcal{Q}_{N}$ for $N \rightarrow \infty$ as follows 7

Identification of the dynamical group and coherent states

The dynamical group $\mathcal{G}_{N}$ is defined as the group generated by the operators $\hat{A}, \hat{B}$ and $\hat{C}$. From (42) we obtain the commutation rules for its Lie algebra $\mathfrak{g}_{N}$

$$
[\hat{A}, \hat{B}]=\frac{2 i}{N} \hat{A},[\hat{A}, \hat{C}]=\frac{i}{N} \hat{B},[\hat{B}, \hat{C}]=\frac{2 i}{N} \hat{C}
$$

that, via the linear transformations

$$
\hat{K}_{0}=\frac{1}{2}(\hat{A}+\hat{C}), \hat{K}_{1}=\frac{1}{2} \hat{B}, \hat{K}_{2}=\frac{1}{2}(\hat{A}-\hat{C}),
$$

become Eqs. (16), with the structure constants consistently rescaled by a factor $1 / N$ (in fact the unscaled rules are realized with the elements $\left.N \hat{K}_{\alpha}\right) \cdot \mathcal{G}_{N}$ can then be regarded as a unitary representation on $\mathcal{H}_{N}$ of the group $\mathcal{G}=S U(1,1)$

nomials of $\mathfrak{g}$. It is possible to demonstrate 15 that the representations of $\mathfrak{g}$ and $U(\mathfrak{g})$ are the same.

7 In order to avoid explicit rescalings of the coupling constants in the Hamiltonian as $N \rightarrow \infty$, a factor $1 / \sqrt{N}$ has been included in the definition of $\hat{q}_{i}$ and $\hat{p}_{i}$, as seen from Eq. (42) 
and the GCS for $\mathcal{Q}_{N}$ are the PCS introduced in Sec. 2.5. For convenience, the indices $k$ (Bargmann index) and $m$, there defined will be rescaled by a factor $N$, i.e. $k \rightarrow N k, m \rightarrow N m$.

Classical operators and equivalent states Using the $\tau$-coordinates introduced at the end of section 2.5, the PCS overlaps [16]

$\left\langle\Omega \mid \Omega^{\prime}\right\rangle_{N}=\frac{\left(1-\left|\tau^{\prime}\right|^{2}\right)^{N k}\left(1-|\tau|^{2}\right)^{N k}}{\left(1-\tau^{\prime} \tau^{*}\right)^{2 N k}}$,

and the matrix elements

$$
\left\{\begin{array}{l}
\frac{\left\langle\Omega\left|\hat{K}_{0}\right| \Omega^{\prime}\right\rangle}{\left\langle\Omega \mid \Omega^{\prime}\right\rangle}=k \frac{1+\tau^{\prime} \tau^{*}}{1-\tau^{\prime} \tau^{*}}, \\
\frac{\left\langle\Omega\left|\hat{K}_{1}\right| \Omega^{\prime}\right\rangle}{\left\langle\Omega \mid \Omega^{\prime}\right\rangle}=2 k \frac{\operatorname{Re} \tau}{1-\tau^{\prime} \tau^{*}}, \\
\frac{\left\langle\Omega\left|\hat{K}_{2}\right| \Omega^{\prime}\right\rangle}{\left\langle\Omega \mid \Omega^{\prime}\right\rangle}=-2 k \frac{\operatorname{Im} \tau}{1-\tau^{\prime} \tau^{*}},
\end{array}\right.
$$

show us that $\hat{K}_{\alpha}$ are all classical operators, in agreement with the definition (33); therefore, as they are basic operators, all $O(N)$-invariant operators are classical. We can obtain Eqs. (46) using the action of $\hat{K}_{ \pm}$on the $|k, m\rangle$-states, defined by Eq. (18),

$$
\begin{array}{lc}
\hat{K}_{ \pm}|k, m\rangle= & \text { a PCS, } \\
=\sqrt{N(k+m)(N(k+m) \pm 1)-N k(N k-1)} \mid k, & \begin{array}{l}
\text { assuming } \\
=1\rangle_{\hat{K}}
\end{array}
\end{array}
$$

and the PCS expansion in terms of $|k, m\rangle$, which in $\tau$-coordinates is 16

$$
\begin{aligned}
|\Omega\rangle= & \left(1-|\tau|^{2}\right)^{N k} . \\
& \cdot \sum_{m=0}^{\infty} \sqrt{\frac{\Gamma(N(2 k+m))}{(N m) ! \Gamma(2 N k)}} \tau^{N m}|k, m\rangle,
\end{aligned}
$$

where $\Gamma$ is the Euler's gamma function. If we now consider Eq. (46) for $\tau^{\prime}=\tau$, i.e.

$$
\left\{\begin{array}{l}
K_{0}(\Omega)=\left\langle\Omega\left|\hat{K}_{0}\right| \Omega\right\rangle=k \frac{1+|\tau|^{2}}{1-|\tau|^{2}} \\
K_{1}(\Omega)=\left\langle\Omega\left|\hat{K}_{1}\right| \Omega\right\rangle=2 k \frac{\operatorname{Re} \tau}{1-|\tau|^{2}} \\
K_{2}(\Omega)=\left\langle\Omega\left|\hat{K}_{2}\right| \Omega\right\rangle=-2 k \frac{\operatorname{Im} \tau}{1-|\tau|^{2}}
\end{array},\right.
$$

we correctly find that the symbols of classical operators are different only for states belonging to different equivalence classes.

\section{Proof of hypothesis}

1) Irreducibility of $\mathcal{G}_{N}$ : This hypothesis needs no proof, as we actually assume it in order to define a consistent quantum theory for any fixed $N$. Notice, in fact, that we have already enforced it when considering the PCS, as we have required the value of the Casimir operator $\hat{K}^{2}=$ $-\hat{K}_{\alpha} \hat{K}^{\alpha}$ on $\mathcal{H}_{N}$ to be fixed (Schur's lemma).

2) Uniqueness of the "Zero" operator: Suppose there exists some operator $\hat{Z}$ for which $Z(\Omega)=$ $\langle\Omega|\hat{Z}| \Omega\rangle=0$ for any PCS $|\Omega\rangle$. Using the commutation relations $\left[\hat{K}_{-}, \hat{K}_{+}\right]=(2 / N) \hat{K}_{0}$ and $\hat{K}_{-}|k, 0\rangle=0$, where $\hat{K}_{ \pm}$and $|k, 0\rangle$ are the shift operators and the reference state, respectively, introduced in Sec. 2.5, one can show by an induction argument 8 , that $\left\langle k, 0\left|\hat{K}_{-} \ldots \hat{K}_{-} \hat{Z} \hat{K}_{+} \ldots \hat{K}_{+}\right| k, 0\right\rangle=0$ for any number of $\hat{K}_{-}$or $\hat{K}_{+}$. Polynomials in $\hat{K}_{+}$applied to $|k, 0\rangle$ clearly form a dense set of $O(N)$-invariant states, then $\hat{Z}$ must be the zero operator.

8 Since $|k, 0\rangle$, as reference state (see Sec. 2.5), is a PCS, $Z(\Omega)=0$ implies $\langle k, 0|\hat{Z}| k, 0\rangle=0$. Then, assuming that

$\left\langle k, 0\left|\hat{K}_{-} \ldots \hat{K}_{-} \hat{Z} \hat{K}_{+} \ldots \hat{K}_{+}\right| k, 0\right\rangle=0$

is true when the total number of $\hat{K}_{-}$plus $\hat{K}_{+}$ is less that $n$, we must prove the same holds when such number becomes $n$. Firstly note that $Z(\Omega)=0$ implies $\left.\langle k, 0|\left[\left[\hat{Z}, \hat{\Lambda}_{1}\right], \hat{\Lambda}_{2}\right], \ldots \hat{\Lambda}_{n}\right]|k, 0\rangle=$ $0 \quad \hat{\Lambda}_{i} \in \hat{K}_{-}, \hat{K}_{+}($choose $|\Omega\rangle=\hat{\Omega}|0\rangle$ with $\hat{\Omega}=$ $\mathrm{e}^{t_{1} \hat{\Lambda}_{1}} \mathrm{e}^{t_{2} \hat{\Lambda}_{2}} \ldots \mathrm{e}^{t_{n} \hat{\Lambda}_{n}}$ and differentiate $Z(\Omega)$ with respect to each $t_{i}$ ). Expanding the multiple commutator, we find that only one term contains all $\hat{K}_{-}$ operators to the left and all $\hat{K}_{+}$operators to the right of $\hat{Z}$. Every other term contains at least one $\hat{K}_{-}$operator which may be pushed right until it annihilates $|k, 0\rangle$, or one $\hat{K}_{+}$operator which may be pushed left. This process produces also commutator terms $\left[\hat{K}_{-}, \hat{K}_{+}\right]$which reduce $n$ by two. In the end the vacuum expectation value of a multiple commutator contains a term of the form (49) with $n$ operators plus lower-order terms which vanish for induction. Therefore Eq. (49) also holds for a number $n$ of $\hat{K}_{-}$plus $\hat{K}_{+}$operators. 
3) Exponential decrease of inequivalent coherent states overlap: This condition easily follows from Eq. (45), implying

$\lim _{N \rightarrow \infty}\left\langle\Omega \mid \Omega^{\prime}\right\rangle_{N}=\mathrm{e}^{-\lim _{N \rightarrow \infty} N \Delta\left(\tau, \tau^{\prime}\right)_{N}}$,

where

$\Delta\left(\tau, \tau^{\prime}\right)_{N}=\Delta\left(\tau, \tau^{\prime}\right)=$

$\left.-k\left[\ln \left(1-\left|\tau^{\prime}\right|^{2}\right)+\ln \left(1-|\tau|^{2}\right)-2 \ln \left(1-\tau^{\prime} \tau^{*}\right)\right)\right]$

so that $\exists \lim _{N \rightarrow \infty} \Delta\left(\tau, \tau^{\prime}\right)_{N} \forall \tau, \tau^{\prime}$ with

$\operatorname{Re} \Delta\left(\tau, \tau^{\prime}\right) \begin{cases}>0 & \text { if }|\Omega\rangle \neq\left|\Omega^{\prime}\right\rangle \\ =0 & \text { if }|\Omega\rangle=\left|\Omega^{\prime}\right\rangle\end{cases}$

4) Classical limit of Hamiltonian: As any $N$ independent polynomial in $\hat{A}, \hat{B}$ and $\hat{C}$ is a classical operator, this holds true also for any Hamiltonian of the form (40).

\section{Classical theory}

We can define a classical dynamics on the coset $S U(1,1) / U(1)$, which is the manifold $P S^{2}$ described in Sec 2.5. that can be mapped to the so-called Poincaré half plane $\mathbb{H}$ by the conformal transformation

$z:=\varrho-i v:=\frac{i+\tau}{i-\tau}$.

$\mathbb{H}$ is endowed with the natural metric

$d s^{2}=\frac{R^{2}}{\varrho^{2}}\left(d \varrho^{2}+d v^{2}\right)$

where $R=\sqrt{k / 2}$, and with the standard Poisson brackets

$\{f, g\}_{P B}=\frac{\partial f}{\partial v} \frac{\partial g}{\partial w}-\frac{\partial g}{\partial v} \frac{\partial f}{\partial w}$,

once $w=k / \varrho$ has been defined. Considering the transformations (44) together with Eq. (48) in the coordinates $(v, w)$, as defined above, we obtain

$$
\left\{\begin{array}{l}
A(v, w)=w \\
B(v, w)=2 v w \\
C(v, w)=w\left(\frac{k^{2}}{w^{2}}+v^{2}\right) .
\end{array}\right.
$$

From Eqs. (38) and (40) we then get the classical Hamiltonian:

$h_{c l}(v, w)=h\left(w, 2 v w, v^{2} w+k^{2} w^{-1}\right)$.

Finally we can define a classical action

$S_{c l}=\int d t\left[v \dot{w}-h_{c l}(v, w)\right]$,

from which the equations of motion of the classical theory, i.e the Hamilton's equations, can be derived

$$
\left\{\begin{array}{l}
\dot{v}=-\frac{\partial h_{c l}}{\partial w}=\left\{h_{c l}, v\right\}_{P B}, \\
\dot{w}=\frac{\partial h_{c l}}{\partial v}=\left\{h_{c l}, w\right\}_{P B} .
\end{array}\right.
$$

\section{Role of the symmetry $O(N)$}

We highlight that in the above construction the role of the global symmetry $O(N)$ is crucial. Indeed had it been absent, the GCS would not have been in one-to-one correspondence with the points of $P S^{2}$, but with those of the much bigger $N$-dimensional complex plane $\mathbb{C}^{N}$. Denoting with $\mathrm{H}_{4}$ the so called Heisenberg group, from which one obtains the standard "Harmonicoscillator" coherent states [8], we can graphically summarize the job done by the symmetry as follows:

$$
\begin{aligned}
& \left|\Omega_{N}\right\rangle \leftrightarrow \quad\left(H_{4} / U^{2}(1)\right)^{N} \simeq \mathbb{C}^{N}, \Omega_{N} \in \mathbb{C}^{N} \\
& \| O(N) \text {-symmetry }
\end{aligned}
$$

$|\Omega\rangle_{N}:=\left[\left|\Omega_{N}\right\rangle\right]_{\sim} \leftrightarrow \frac{S U(1,1)}{U(1)} \simeq P S^{2}, \Omega \in P S^{2}$

where the equivalence relation is constructed thanks to the symmetry transformations, as in Eq. (39).

4.1 Quantum-to-classical crossover of angular momentum

Thanks to Eqs. (37) and (48) we can calculate the expectation value on PCS of the Casimir 
operator $\hat{K}^{2}=-\hat{K}_{\alpha} \hat{K}^{\alpha}$ :

$K^{2}(v, w)=k^{2}$.

Notice that this is constant, i.e. it does not depend on the conjugated variables $(v, w)$. This means that it has to flow to a conserved quantity in the classical motion. It is then a due question to ask: "which one?". It is suggestive to analyze the connection of $\hat{K}^{2}$ with the $N$ degrees of freedom. After some calculations we obtain:

$\hat{K}^{2}=\frac{1}{4}\left(\hat{L}^{2}+\frac{1}{4}-\frac{1}{N}\right)$,

where

$$
\left\{\begin{array}{l}
\hat{L}^{2}=\frac{1}{2} \sum_{i j} \hat{L}_{i j}^{2} \quad i, j=1, \ldots, N, \\
\hat{L}_{i j}=\hat{q}_{i} \hat{p}_{j}-\hat{q}_{j} \hat{p}_{i},
\end{array}\right.
$$

are the modulus and the components of the angular momentum, respectively, of the $N$ degrees of freedom. Then the above mentioned conserved classical quantity might be an angular momentum. Such an identification is reinforced by the following argument:

- i) If we express the positions $\hat{q}_{i}$ and the momenta $\hat{p}_{i}$ in terms of the ladder operators $\left(\hat{a}_{i}, \hat{a}_{i}^{\dagger}\right):$

$$
\left\{\begin{array}{l}
\hat{q}_{i}=\frac{1}{\sqrt{2}}\left(\hat{a}_{i}^{\dagger}+\hat{a}_{i}\right) \\
\hat{p}_{i}=\frac{i}{\sqrt{2}}\left(\hat{a}_{i}^{\dagger}-\hat{a}_{i}\right)
\end{array}\right.
$$

the canonical commutation rules (42) become

$$
\left[\hat{a}_{i}, \hat{a}_{j}^{\dagger}\right]=\frac{1}{N} \delta_{i j} \hat{\mathbb{I}} \text { with } i, j=1, \ldots, N
$$

and thanks to Eqs. (41) and (44) it is:

$$
\hat{K}_{0}=\frac{1}{2}\left(\hat{\mathcal{N}}+\frac{1}{2}\right)
$$

where $\hat{\mathcal{N}}=\sum_{i} \hat{a}_{i}^{\dagger} \hat{a}_{i}$ is the number operator.
- ii) As it is well known, the relations 63 imply that the spectrum of $\hat{\mathcal{N}}$ is the set $\mathbb{N}$ of the natural numbers and then, according to Eq. (64), the spectrum of $\hat{K}_{0}$ is $\left\{\frac{1}{2}\left(n+\frac{1}{2}\right)\right.$, $n \in \mathbb{N}\}$. Defining $n:=l+2 m$ with $l, m \in$ $\mathbb{N}$, and considering Eqs. (18), the possible values of $k$ must be

$k=\frac{\tilde{l}}{2}:=\frac{1}{2}\left(l+\frac{1}{2}\right) \quad$ with $l \in \mathbb{N} ;$

in fact, a more exhaustive demonstration of Eq. (65) can be found in Ref. [17.

- iii) The physical meaning of the natural number $l$ is revealed when inserting Eq. (65) in Eq. (18) to see that, using Eq. (60), it is 9

$$
\begin{aligned}
& \hat{L}^{2}|l, m\rangle=l\left(l+1-\frac{2}{N}\right)|l, m\rangle \\
& \stackrel{N \rightarrow \infty}{\longrightarrow} \\
& \hat{L}^{2}|l, m\rangle=l(l+1)|l, m\rangle \quad l \in \mathbb{N}
\end{aligned}
$$

where we have written the eigenstates $|k, m\rangle$ as $|l, m\rangle$. Eq. (66) shows that, if the limit $N \rightarrow \infty$ is performed, the operator $\hat{L}^{2}=$ $4 \hat{K}^{2}-\frac{1}{4}$ has the same spectrum of the modulus of a 3-dimensional orbital angular momentum operator.

- iv) Finally inserting Eq. (65) in Eq. (59) it is

$4 K^{2}(v, w)=4 k^{2}=\tilde{l}^{2} ;$

it is hence appropriate to assume that the quantity $4 k^{2}$ flows to a classical $3 \mathrm{~d}$ angular momentum, conserved in the motion. Moreover the dependence on $\tilde{l}=l+\frac{1}{2}$ confirms that the limit $N \rightarrow \infty$ is a classical one 10

9 Notice that, considering the rescaling of the $S U(1,1)$ commutation rules and of the indices $k, m$, Eq. (18) assumes the form:

$\left\{\begin{array}{l}N^{2} \hat{K}^{2}|k, m\rangle=N k(N k-1)|k, m\rangle \\ N \hat{K}_{0}|k, m\rangle=N(k+m)|k, m\rangle\end{array}\right.$

10 The classical limit of a spin- $j$ system can be naively implemented substituting the spin operators 
In the end, a "bridge" from "quantum to classical" is built thanks to the three real parameters $(v, w, k)$ that have a genuine quantum origin but, in the limit $N \rightarrow \infty$, entering in the Hamiltonian (56), do acquire a proper classical nature. In particular, from the quantum viewpoint, the Bargmann index $k$ identifies the theory's Hilbert space $\mathcal{H}_{N}$ as an irreducible representation of $S U(1,1)$ and $(v, w)$ the overcomplete set of PCS. As a result of the crossover to the classical theory, $(v, w)$ become the conjugated variables defining the motion, and $4 k^{2}$ is a conserved angular momentum.

Despite the above, quite convincing, discussion, there is a caveat: the action (57) tells us that the classical motion is 1-dimensional, implying that no angular momentum can be defined. The only possibility is hence that such an angular momentum is external to the system. In fact, noticing that $w$ has to be positive 11, it can be mapped into a radius $r$; therefore, we suggest that the emerging classical theory describes the central motion of a 3-dimensional particle in the 1-dimensional effective-potential formalism, with respect to the radial coordinate $r$. In the next subsection we show how this statement is substantiated.

\subsection{A paradigmatic example: the free particles}

Let us use the above designed procedure to find the classical limit of a quantum theory that describes a number $N \rightarrow \infty$ of one-dimensional distinguishable free particles. The quantum Hamiltonian is:

$\hat{H}_{N}=\frac{N}{2} \sum_{i} \hat{p}_{i}^{2}=N \hat{C}$.

with classical vectors that freely move on a sphere of radius $(j+1 / 2)$ [18.

11 This is easily proved by noticing from Eq. (25) that $|\tau|^{2}<1$, and using the transformation Eq.(52) and the one after Eq. (54)
The classical phase space is $\mathbb{H}$ with the two coordinates $(v, w)$; the classical Hamiltonian describing the limit $N \rightarrow \infty$ is, according to Eq. (56),

$h_{c l}(v, w)=v^{2} w+k^{2} w^{-1}$.

If we relabel $w=r^{2} / 2, v=p / r$, then $\{p, r\}_{P B}=$ 1 and Eq. (69) becomes

$h_{c l}(p, r)=\frac{p^{2}}{2}+\frac{\tilde{l}^{2}}{2 r^{2}}$.

This is indeed the Hamiltonian of a classical 3-d free particle with angular momentum $L^{2}=\tilde{l}^{2}$ in the effective potential formalism.

We have thus obtained that a large number $N$ of quantum free particles corresponds to one single classical rotating particle. It is of great relevance and significance that one cannot recover the quantum Hamiltonian (68) from the classical one (70) simply substituting the dynamical variables $p^{2}$ and $r^{2}$ with the operators $\hat{p}^{2}=\sum_{i}{\hat{p_{i}}}^{2}$ and $\hat{r}^{2}=\sum_{i} \hat{q}_{i}{ }^{2}$, and imposing the rules $i[\hat{p}, \hat{r}]=\hat{\mathbb{I}}$ i.e. by a naive "quantization": indeed the classical limit of quantum theory is most often a completely different theory.

\section{Conclusions}

Before presenting our concluding remarks, let us briefly comment upon the difference between the quantum to classical crossover of a theory, which is the topic to which this work is dedicated, and the suppression of quantum features in the behaviour of non-isolated quantum systems, either closed or open 12. The two processes

12 Despite the ambiguity of the terminology, in recent literature "closed" systems are defined as quantum non-isolated systems with an environment that enters the analysis as a classical-like agent, such as an external magnetic field, or a classical thermal bath. "Open" quantum systems, instead, are those whose environment is, and must be treated as, a quantum system, which implies having to consider phenomena such as entanglement generation, back-flow of information, non-markovianity, and many others. 
are of a profoundly different nature. The former occurs whenever the system that the theory describes is "big" (i.e. made of a very large number of components) and features some global symmetry, irrespective of the possible presence of other systems in the overall setting. It is a process that changes the theoretical framework into which the description of the system is set. The latter, instead, is observed in quantum systems WITH an environment, and the loss of quantum features is a direct consequence of such an environment being "big" in the above sense. In this case, the principal system stays "quantum" (i.e. described by the formal tools of quantum mechanics), and it can possibly recover its quantum features. In fact, it has been recently shown [19, 4] that the loss of quantum features in systems that interact with environments made by a very large number of components can be formally derived and related with the theory of quantum measurements, once the environment undergoes a quantum to classical crossover as described by the first process. In brief, one can say that the quantum to classical crossover is defined as a process "per sé", while the loss of quantum features in a system is a consequence of one such crossover occuring in the environment.

We finally get to our Conclusions, and start by interpreting the results of the previous section from a different viewpoint. Consider a quantum theory $\mathcal{Q}_{\chi}$ that describes a spinless particle in 1-dimension, and depends on some "quanticity" parameter $\chi$, say a coupling constant. Its dynamical group is $H_{4}$, that can be identified with $\mathcal{G}_{N=1} 13$ Noting that $H_{4} \simeq \mathcal{G}_{N}$ (they are both representations of $\mathcal{G}$ ), we find that there exists a correspondence between the GCS $|\Omega\rangle_{\chi}$ of $Q_{\chi}$ and the GCS $\left|\Omega_{N}\right\rangle$ of $\mathcal{Q}_{N}$, i.e. one can write $\Omega=\Omega\left(\Omega_{N}\right)$. Then, given the quantum Hamiltonian $\hat{H}_{\chi}$ of $\mathcal{Q}_{\chi}$, we can find a Hamilto-

13 This result is clear when considering Eqs. 63) for $N=1$. nian $h$ of the form (40) such that

$H_{\chi}\left(\Omega\left(\Omega_{N}\right)\right)=N h\left[A\left(\Omega_{N}\right), B\left(\Omega_{N}\right), C\left(\Omega_{N}\right)\right]$.

Therefore, when $N \rightarrow \infty$ we obtain a classical theory not only for $\mathcal{Q}_{N}$ but also for $\mathcal{Q}_{\chi}$, possibly when $\chi \rightarrow 0$. This argument can be generalized to the $N \rightarrow \infty$ limit of essentially all physical quantum theory equipped with a global symmetry 14

One may thus presume that any system we perceive as classical is in fact a particular macroscopic quantum system, of which we observe the effective behaviour. The explicit implementation of Yaffe's procedure given above for a few paradigmatic physical systems, clearly illustrate some inherent properties of the quantum to classical crossover in the macroscopic limit, which are worth to be highlighted. Among them, probably one of the most apparent is that many different quantum theories can flow into the same classical theory, whose Hamiltonian may appear rather different from the one expected by doing a naïve classical limit of the quantum one. However, the one outcome upon which we would like to comment the most, is that the classical theory we finally get by the present formal approach is not that whose conventional quantization would lead to the quantum theory from which we started: This is, in our opinion, an especially relevant observation, as it tells us that apparently the proper way of reasoning is that of moving from the quantum to the classical description, and not that of quantizing classical theories. We think that such change of perspective could be fruitful to address some still unsolved problems, as, e.g., a proper quantum description of gravitation.

14 Yaffe demonstrates that not only $O(N)$ vector models have a classical limit when $N \rightarrow \infty$, but also $U(N)$ matrix models and $U(N)$-lattice gauge theories. 
Acknowledgements Financial support from the Università degli Studi di Firenze in the framework of the University Strategic Project Program 2015 (project BRS00215) is gratefully acknowledged. PV has worked in the framework of the Convenzione Operativa between the Institute for Complex Systems of the Italian National Research Council (CNR) and the Physics and Astronomy Department of the University of Florence.

\section{Conflict of interest}

The authors declare that they have no conflict of interest.

\section{References}

1. L. Yaffe, Rev. Mod. Phys. 54, 407 (1982)

2. P. Liuzzo-Scorpo, A. Cuccoli, P. Verrucchi, EPL (Europhysics Letters) 111(4), 40008 (2015)

3. M.A.C. Rossi, C. Foti, A. Cuccoli, J. Trapani, P. Verrucchi, M.G.A. Paris, Phys. Rev. A 96, 032116 (2017)

4. C. Foti, T. Heinosaari, S. Maniscalco, P. Verrucchi, Quantum 3, 179 (2019)

5. A. Coppo, Schwarzschild Black Holes as Macroscopic Quantum Systems (Master Thesis, Università degli studi di Firenze, 2019)

6. R. Gilmore, Ann. Phys. 74, 391 (1972)

7. A. Perelomov, Commun. Math. Phys. 26, 222 (1972)

8. W. Zhang, R. Gilmore, H. Feng, Rev. Mod. Phys. 62, 867 (1990)

9. J. Lee, Introduction to Smooth Manifolds (Springer, 2012)

10. L. Hua, Translations of Mathematical Monographs 6 (1963)

11. V. Bargmann, Ann. Math. 48, 568 (1947)

12. L. Biedenharn, J. Nuyts, N. Straumann, Ann. Inst. Henri Poincaré 3, 13 (1965)

13. A. Barut, C. Fronsdal, Proc. R. Soc. Lond. A 287, $532(1965)$

14. A. Perelomov, Generalized Coherent States and Their Applications (Springer-Verlag, 1985)

15. A. Barut, R. Raczka, Theory of Group Representations and Applications (Polish Scientific Publishers, 1980)

16. M. Novaes, Revista Brasileira de Ensino de Fisica 26, 351 (2004)

17. T. Friedmann, C. Hagen, J. Math. Phys. 53, $122102(2012)$

18. E. Lieb, Commun. Math. Phys. 31, 327 (1973)

19. F.G.S.L. Brandao, M. Piani, P. Horodecki, Nat. Comm. 6, 7908 (2015) 